\title{
The Anatomy of a Geometric Algorithm*
}

\author{
Jiří Matoušek \\ Department of Applied Mathematics, Charles University \\ Prague, Czech Republic \\ matousek@kam.ms.mff.cuni.cz
}

\section{What is computational geometry?}

(examples rather than a definition)

An $n$-point set in $\mathbf{R}^{3}$ :

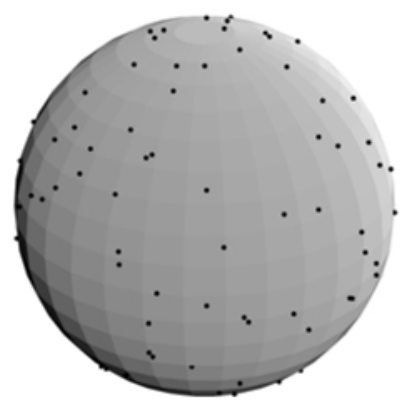

- What is the diameter of this point set?

- What is the smallest ball enclosing it?

- What is the "best fit" by a sphere?

- What is a "nice surface" defined by these points?

^ This article contains the speaker's slides for the talk, reproduced by the editors with speaker's permission. An appendix with references was prepared by the speaker. 


\section{OUR PARTICULAR}

\section{PROBL-FA4 • •}

INPUT! AX POINTS, in the plane , $m$

$F \mid M): \quad$ A SMAШEST ClftCIf ke PoInTs

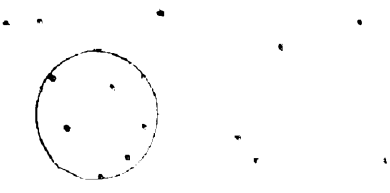

TRICK. $\cdots \quad$ I-.0OK AT

\section{FIXED SIZE WO 6LEM}

ginen $m$ points and $r \geq 0$, is theres a circle

of radius on clising

5? 4 (seitt'ts ${ }^{3}$.

\footnotetext{
${ }^{11}$ TestINS" gincen $n$

$S$ "Conversion" B.

$\{$ generat TECHNiques

* (PARAHETRÍ 5EAMH \& OTHERS)

"CEACenine" for min $\{n \geqslant 0$ : test positive
}

WHIL DISCLISSAN AN ALEOB.TTHM | WE WU, (MEPT

ft \&E-'Vt.:Ti.ftL, Fi Wt ${ }^{-} \quad$ "ETCNV

- Reformeladion in terms of arba., g g e $\mathrm{R}^{\mathrm{R}} *$ \&cs

- Nsirla combinaterial. geometric observations

- rondomized algoithms

barametric search calle hol*i "ks o,voie( i:*)

- "n"-havel" problems

- geomethic optimization tre bin «WOV K (L.P. type nublews)

- tVpical obstacleg to IMPLE $>$ E NTATION
R E F o R fM.ut.AT: ${ }^{1}$ ONJ

WITH ARRANEEMENTS

3 U-diSC $<$ SIAt£Wviq $\geqslant$ ?

$$
\Longleftrightarrow
$$

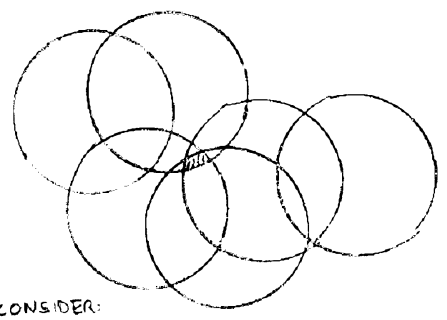

CONSIDER

$n$ diste of radius $r$

around the gin points

$\exists$ point of DEDTH $\geqslant k$

depth $=$ \# of containing dises ) 


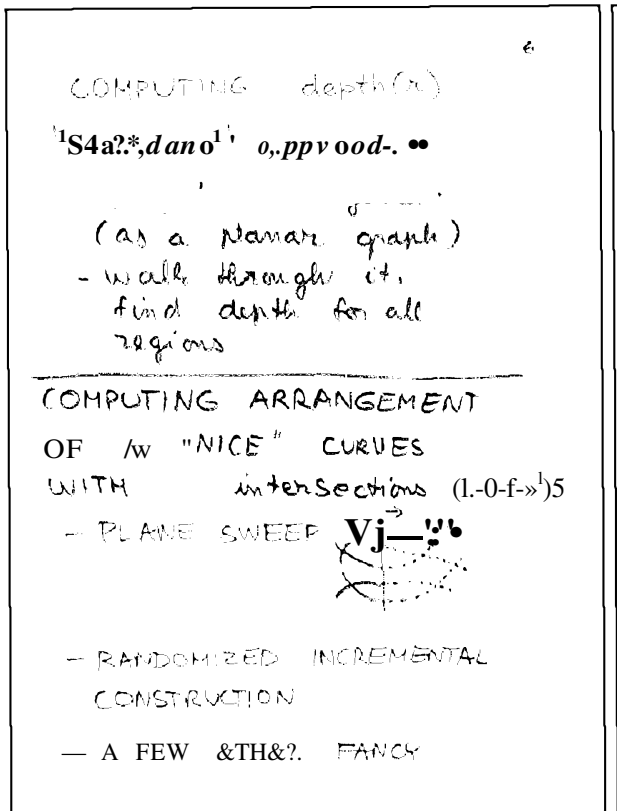

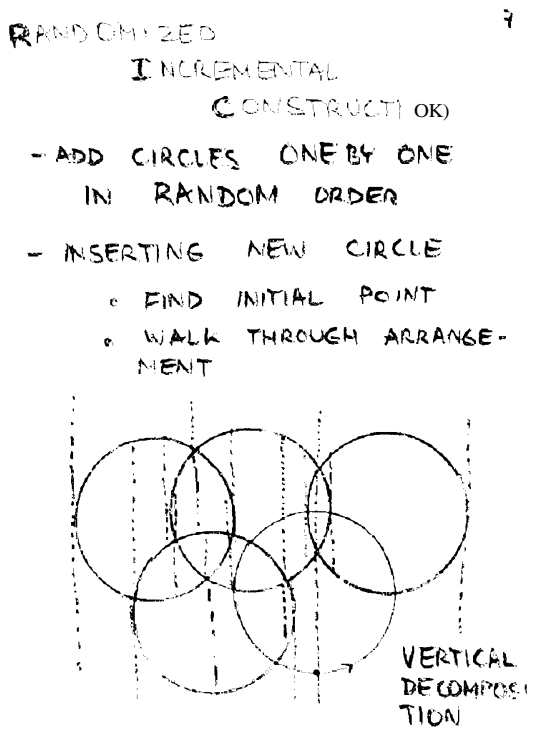

COHfi!MATORIAL INFORMATION:

IP «\%Ji,fX) 》£

TURK:

(fF 1

Oil v«

$\mathrm{DM}^{\mathrm{*}}$

EygM MOE.E IS TRUE :

IF MEN

EACH DISC IM7£《SEXTS, IOST $\theta(t)$ OWests

fümortant... All havei B SAME RADIUS! 3

nith

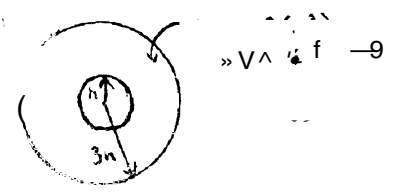




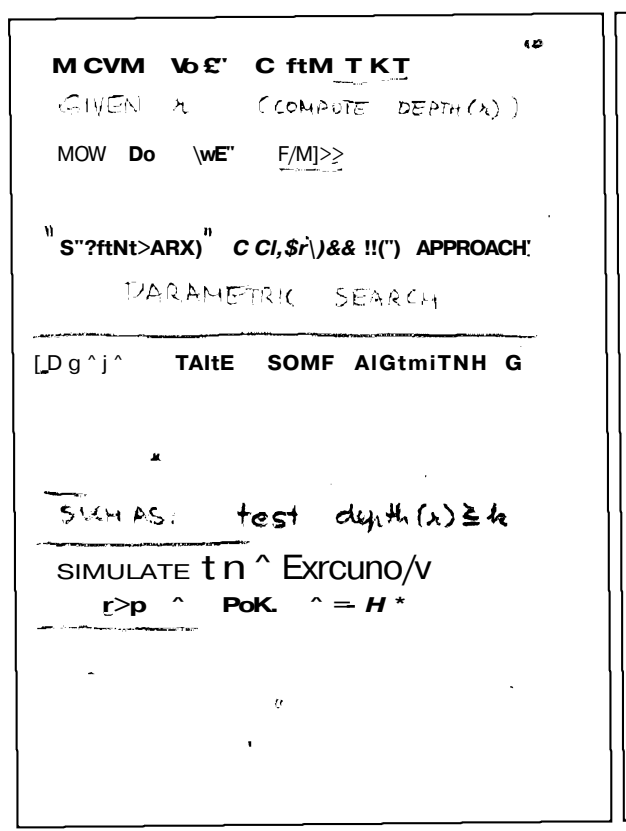
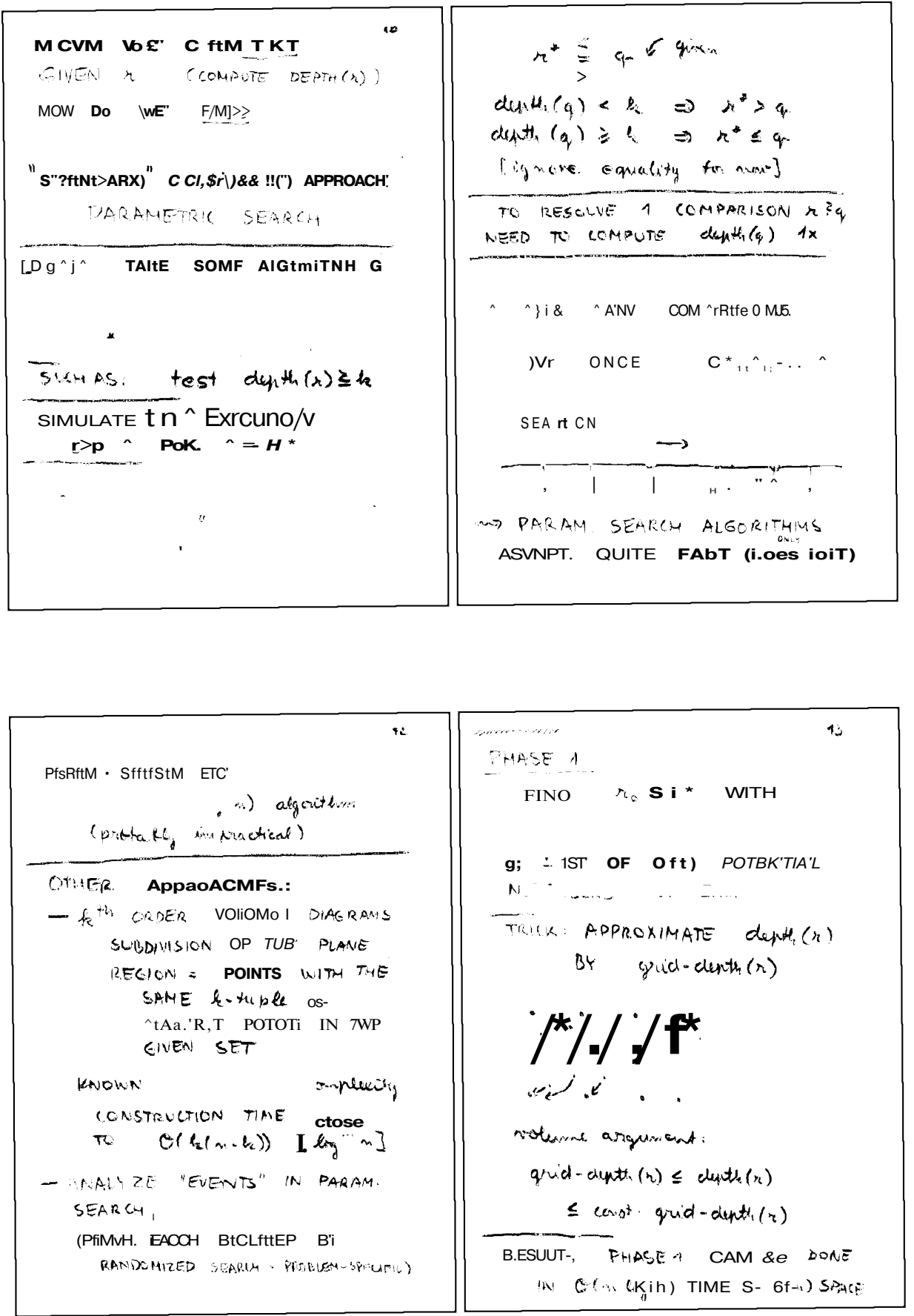


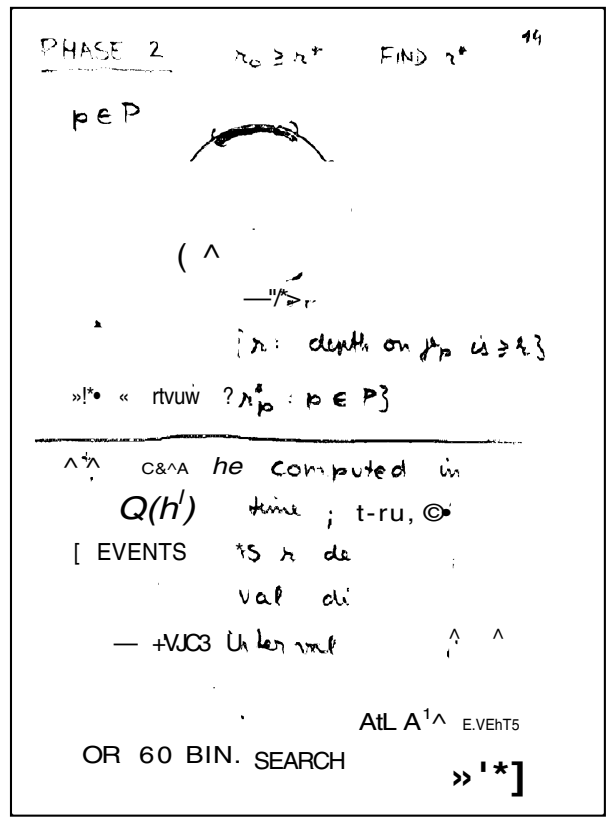

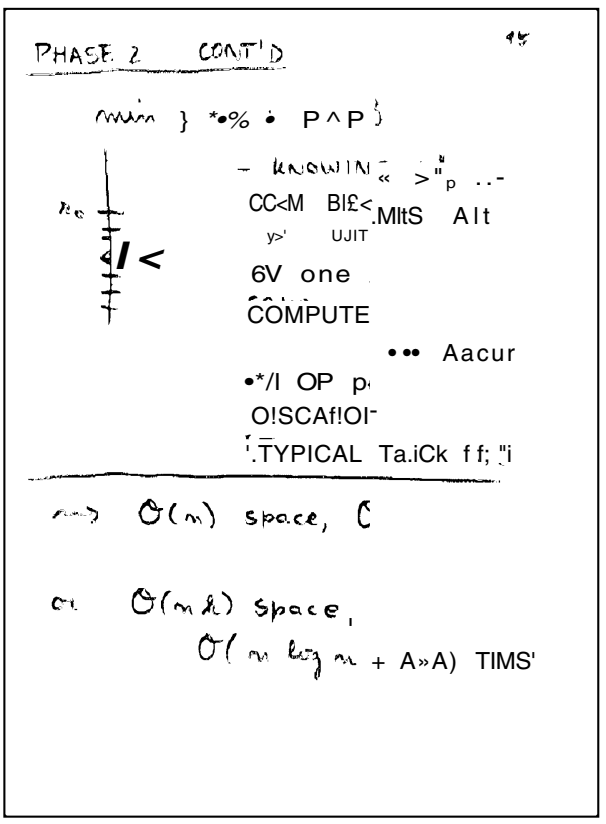

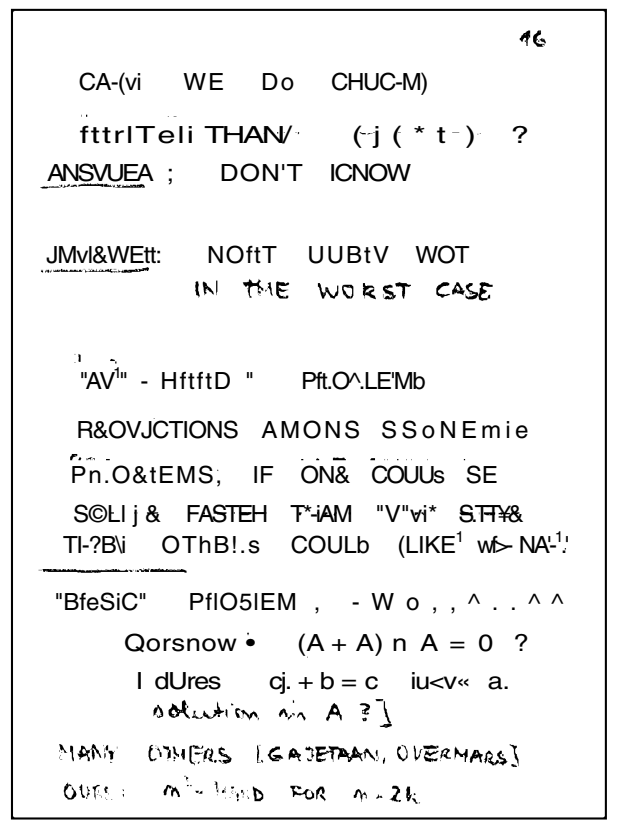

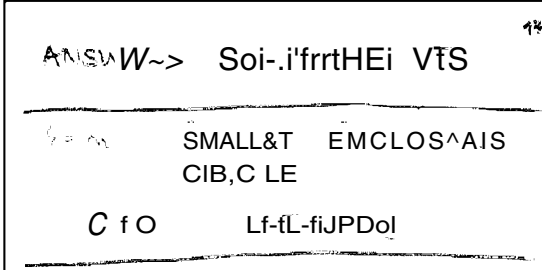

EEMFYNi,I_ Ff2. AMEW OfiK:

1. SHArtIR1tMEi_2.LI

"ABST"HACT" OPTIMI2ATIOIII PRO6LC/M AXIOM^ \&• PRIMITIVE $\mathrm{O}^{\wedge}$ eaAriows

CMECK (VXIOMS g< iMfteWEivr

räIITIVS ÖPËfl-ATIOIVi

$\therefore>$ SEu^riAL Pew EftFuy At\&o^ITViMS

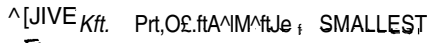

ENC LOSIWG. $g, t, L t \quad I M \wedge$ pISTB,IMCE OT ^O^OUVHBOAA! ĆOŇVEit 
IMPLE'M`'TAtT|OM

\section{k. OosfT}

$<$ ? $^{*}<{ }^{*}-$ ? $\quad$ SHALL

LP-TVPE- Pp-ti \& LWH Ui TTM

9 WULATED CONS7R.A-'N7\&

$$
O\left(M \log , M+a_{i} *, J\right.
$$

( HOT (VET) PRACTICAL •)

ey. cwc3

$\therefore=2$ Provably $\Omega(n \log m)$

$a=0 \quad \sigma\left(x_{1}\right)$
- $1 \mathrm{H} 1.0 \mathrm{ft}, \mathrm{pT} \mid \mathrm{Cftt}, \quad$ PWHR\& OFTBN < fCVJT F «vmtv DIFFICULT TO PHoCRaA \& USE

- U US ${ }^{\wedge} ; W . V, V, \quad$ COMSH>BRA£.!,g SA)0«k

TC INPLgfHCAJT TH^OS, A,L60 V-JELL - HAWW THINSS WEEO

- NliM^ftiCM. / P»!.rciS5orli tssufii, .

" PI.OftTIMG PoiiVT AR.ITH, WO_gTtV No 6\&O\&

- USE EXACT Aiirrvi. ANB/O/? MMTH, PILT\&S.\& (.LIm^iCi) - S SLo VVER gor p^Vi @pc(aETTE'f? SLOIV TWA Cft- ${ }^{*} \& H I A r{ }^{\wedge}$ 


\section{Appendix}

The purpose of this small appendix to the slides is to cite sources for the results directly mentioned in the talk, and to point to a few general-purpose references on computational geometry.

There are several introductory textbooks by now; one of them is [2]. Randomized incremental algorithms and applications of arrangements can be found there. There are two handbooks of computational geometry [6], [11]. Recent activity in the field, with increasing emphasis on more practically oriented studies, can be monitored in proceedings of the Annual ACM Symposia on Computational Geometry.

A parametric search algorithm for the considered problem is from [3]. Parametric search was formulated in [10]. Other papers on the problem are [4], [1]. The algorithm discussed in some detail is from [9]. The $N^{2}$-hard problems are collected in [5]. The LP-type problems were introduced in [8], and the application on circles enclosing all but $q$ points is in [7].

\section{References}

1. A. Datta, H.-P. Lenhof, C. Schwarz, and M. Smid. Static and dynamic algorithms for $k$-point clustering problems. In Proc. 3rd Workshop Algorithms Data Struct., volume 709 of Lecture Notes Comput. Sci., pages 265-276. Springer-Verlag, 1993.

2. M. de Berg, M. van Kreveld, M. Overmars, and O. Schwarzkopf. Computational Geometry: Algorithms and Applications. Springer-Verlag, Berlin, 1997.

3. A. Efrat, M. Sharir, and A. Ziv. Computing the smallest $k$-enclosing circle and related problems. Comput. Geom. Theor. Appl., 4:119-136, 1994.

4. D. Eppstein and J. Erickson. Iterated nearest neighbors and finding minimal polytopes. In Proc. 4th ACM-SIAM Sympos. Discrete Algorithms, pages 64-73, 1993.

5. A. Gajentaan and M. H. Overmars. On a class of $n^{2}$-hard problems in computational geometry. Comput. Geom. Theor. Appl., 5(3):117-142, 1995.

6. J. E. Goodman and J. O'Rourke, editors. Handbook of Discrete and Computational Geometry. CRC Press LLC, Boca Raton, FL, 1997.

7. J. Matoušek. On geometric optimization with few violated constraints. Discrete Comput. Geom., 14:365-384, 1995.

8. J. Matoušek, M. Sharir, and E. Welzl. A subexponential bound for linear programming. Algoritmica, 16:498-516, 1996.

9. J. Matoušek. On enclosing $k$ points by a circle. Information Processing Letters, 53:217-221, 1995.

10. N. Megiddo. Applying parallel computation algorithms in the design of serial algorithms. Journal of the ACM, 30:852-865, 1983.

11. J.-R. Sack and J. Urrutia, editors. Handbook on Computational Geometry. NorthHolland, 1999. In press. 